\title{
Briófitas do arboreto do Jardim Botânico do Rio de Janeiro*
}

\author{
Lianna de Castro Molinaro ${ }^{1}$ \\ Denise Pinheiro da Costa ${ }^{2}$
}

\begin{abstract}
RESUMO
O trabalho apresenta os resultados do levantamento das espécies de briófitas do Arboreto do Instituto de Pesquisas Jardim Botânico do Rio de Janeiro. Foram identificados 98 táxons (1 antóceros, 40 hepáticas e 57 musgos), distribuídos em 61 gêneros e 37 famílias, existindo um predomínio de musgos $(58 \%)$ sobre hepáticas $(41 \%)$. Seis formas de vida foram caracterizadas (coxim, pendente, taloso, tapete, trama e tufo), predominando tufo, trama e tapete (85\%). Cinco tipos de substrato são colonizados (corticícola, epífila, epíxila, rupícola, terrícola), prevalecendo o corticícola (36\%). Sete padrões de distribuição geográfica foram caracterizados (Cosmopolita, Pantropical, Neotrópico e África, América Tropical e Subtropical, Neotropical, Disjunto e restrito ao Brasil), predominando o Neotropical (46\%). Bryum pseudocapillare, Calymperes tenerum, Ceratolejeunea laetefusca, Cololejeunea minutissima subsp. myriocarpa e Fissidens submarginatus são ocorrências novas para o Rio de Janeiro. Bryum pseudocapillare, Bryum renauldii e Calymperes tenerum são citadas pela segunda vez para o Brasil. Os dados foram comparados com aqueles obtidos para outros Jardins Botânicos e mata atlântica de baixada. Os resultados estão de acordo com os encontrados nas florestas secundárias de terra baixa ou urbanas, que são mais secas e abertas, e sofrem influência direta da poluição, temperatura e luminosidade altas.
\end{abstract}

Palavras-chave: briófitas, Arboreto, Jardim Botânico, Rio de Janeiro.

\begin{abstract}
A floristic study of the bryophytes was carried out in the park of the Rio de Janeiro Botanical Garden. Ninety eight taxa of bryophytes were found (1 anthocerote, 40 hepatics and 57 mosses), in 61 genera and 37 families, predominating more mosses (58\%) than hepatics (41\%). Six life-forms were found (cushion, pendent, thallose, carpet, mat, turf), the most common are turf, carpet, and mat $(85 \%)$. Five kinds of substrates were colonized (corticolous, epiphyllous, epixylous, saxicolous, terricolous), predominating the corticicolous (36\%). Seven distribution patterns were characterized (Cosmopolitan, Pantropical, Neotropical and Africa, Tropical and Subtropical America, Neotropical, Disjunct, and restrict to Brazil), the most common being Neotropical (46\%). Bryum pseudocapillare, Calymperes tenerum, Ceratolejeunea laetefusca, Cololejeunea minutissima subsp. myriocarpa and Fissidens submarginatus are new records to Rio de Janeiro. Bryum pseudocapillare, Bryum renauldii, and Calymperes tenerum are recorded by the second time to Brazil. The data were compared to those found in other Botanical Gardens and in lowland atlantic rainforest. The results are similar to those found in secondary lowland rainforests or in urban areas, that are dry and open areas, suffering directly influence of the air pollution, high temperatures and light intensities.
\end{abstract} Keywords: bryophytes, Arboreto, Botanic Garden, Rio de Janeiro.

\footnotetext{
* Monografia a ser apresentada ao Curso de Ciências Biológicas, Universidade Federal Rural do Rio de Janeiro. ${ }^{1}$ Bolsista de Iniciação Científica, Jardim Botânico do Rio de Janeiro (PIBIC/CNPQ).

${ }^{2}$ Pesquisador Titular, Jardim Botânico do Rio de Janeiro, Rua Pacheco Leão 915, Cep 22460-030, Rio de Janeiro, Brasil, e-mail: dcosta@jbrj.gov.br
} 


\section{INTRODUÇÃO}

O Arboreto do Jardim Botânico, situa-se na cidade do Rio de Janeiro, entre os paralelos $22^{\circ} 58^{\prime} 14$ "'S e 4313'18"W, ocupando uma área de ca. 54 hectares, atravessado pelo Rio dos Macacos, que abastece os lagos, canais e canaletas de todo o parque florístico, com solos aluviais distróficos e eutróficos, textura média e argilosa e clima tropical úmido chuvoso, sendo dezembro, janeiro e fevereiro os meses mais chuvosos (Index Seminum, 1990).

É um parque que abriga espécies brasileiras e exóticas de inestimável valor científico, com representação dos ecossistemas brasileiros e alguns estrangeiros. No total o Arboreto contém 122 aléias, 40 seções, 194 canteiros, 6 estufas e viveiros, com ca. de 8.200 espécies e 40.000 exemplares (S. Iamamoto - comunicação pessoal).

Os Jardins Botânicos contribuem para a conservação dos recursos vivos, mantendo os processos ecológicos e os sistemas vitais essenciais, preservando a diversidade genética e assegurando a utilização sustentável das espécies e dos ecossistemas. Segundo Heywood (1990), é objetivo de um Jardim Botânico elaborar um inventário das plantas de suas reservas e publicar os resultados.

No que se refere a composição florística do parque, desde 1999 vem sendo realizado o Projeto de Inventário e Identificação das Coleções Botânicas e Históricas do Arboreto do Instituto de Pesquisa Jardim Botânico do Rio de Janeiro, com as fanerógamas, sendo a brioflora totalmente desconhecida.

No Brasil poucos são os trabalhos sobre a brioflora de florestas secundárias, de áreas urbanas ou degradadas (Bastos \& Yano, 1993; Costa, 1999b; Rebelo et al., 1995; Hirai et al., 1998).

Segundo Brown \& Lugo (1990), diversas razões justificam a importância do estudo da flora de florestas secundárias nas regiões tropicais, principalmente devido ao aumento deste tipo de formação na região. Em muitos aspectos as florestas secundárias fornecem condições que melhoram os solos e a qualidade da água ou auxiliam na conservação do material genético, dos nutrientes, da umidade e matéria orgânica do solo. Todos estes aspectos são de grande importância para a conservação da biodiversidade nas regiões tropicais.

O presente trabalho tem por objetivo contribuir para o conhecimento da brioflora do Arboreto do Jardim Botânico do Rio de Janeiro, como uma colaboração pioneira, sendo o segundo estudo realizado no Brasil com briófitas de Jardins Botânicos. Assim como, dar continuidade ao inventário das plantas do parque (Projeto Inventário e Identificação das Coleções Botânicas e Históricas do Arboreto do Instituto de Pesquisa Jardim Botânico do Rio de Janeiro), fornecendo dados sobre a brioflora de áreas plantadas em regiões urbanas.

\section{MATERIAL E MÉTODOS}

Entre agosto de 1999 e fevereiro de 2001, foram realizadas 39 excursões para coleta de material briofítico na área do Arboreto do Jardim Botânico, sendo uma destinada a coleta nas copas das árvores. Também foram estudados os exemplares depositados no acervo do herbário RB, coletados entre 1923-1927. Todas essas amostras foram checadas, algumas auxiliaram na identificação dos exemplares coletados e poucas necessitaram de identificação.

A técnica de coleta, herborização e preservação do material botânico, segue Yano (1984b).

A classificação adotada é a proposta por Vitt (1984) para a Divisão Bryophyta, por Schuster (1980) para a Divisão Hepatophyta e Hässel de Menéndez (1988) para a Divisão Anthocerotophyta.

Os resultados são apresentados abrangendo as seguintes análises: composição florística; formas de vida; tipos de substrato; distribuição geográfica; riqueza florística. $\mathrm{Na}$ tabela 1, os táxons estão ordenados por divisão taxonômica, em ordem alfabética de família, gênero e espécie e para cada táxon, são fornecidos dados sobre forma de vida, tipo de 
substrato, variação altitudinal no Brasil e distribuição geográfica no Brasil e no mundo.

A nomenclatura adotada para os tipos de substrato segue a caracterização feita por Robbins (1952).

A classificação adotada para as formas de vida segue a de Mägdefrau (1982), com modificações feitas por Richards (1984).

A distribuição geográfica das espécies no Brasil e no mundo foi baseada nos trabalhos de: Alves (1992); Bastos (2000); Bastos \& Bôas-Bastos (1998, 2000); Bastos et al. (1998a, 1998b, 2000); Behar et al. (1992); Bischler (1964, 1967, 1984); Bôas \& Bastos (1998); Bonner (1953); Bononi (1989); Buck (1998); Costa (1992, 1994, 1999a,); Costa \& Yano (1988, 1993, 1995, 1998); Evans (1925); Florschütz (1964); Florschütz de - Waard (1986); Fulford (1945, 1976); Germano \& Pôrto (1996); Giancotti \& Vital (1989); Gradstein (1981, 1994); Gradstein \& Buskes (1985); Gradstein et al. (1992); Hässel de Menéndez (1961, 1989); Harley (1995); Hell (1969); Herzog (1925); Jovet-Ast (1993); Lemos-Michel (1980, 1983, 1999); Lisboa (1994); Lisboa \& Ilkiu-Borges (1995, 1997); Lisboa \& Maciel (1994); Lisboa \& Yano (1987); Lisboa et al. (1998); Martins et al. (1990); Oliveira (2001); Oliveira e Silva (1998); Pôrto (1990); Pôrto \& Bezerra (1996); Pôrto \& Yano (1998); Pôrto et al. (1999); Pôrto \& Oliveira (2000); Reiner-Drehwald \& Goda (2000); Sá \& Pôrto (1996); Sampaio (1916); Santiago (1997); Schäfer-Verwimp (1989, 1991; 1996); Schäfer-Verwimp \& Giancotti (1993); Schäfer-Verwimp \& Vital (1989); Schiffner \& Arnell (1964); Sharp et al. (1994); Spruce (1884-1885); Stotler (1969); Stotler et al.(1999); Vianna (1970, 1976, 1981, 1985); Visnadi (1998), Visnadi \& Vital (1989); Vital et al. (1991); Vital \& Visnadi (1994); Yano (1984a, 1987, 1989, 1993, 1994, 1995, 1996); Yano \& Andrade-Lima (1987); Yano \& Colletes (2000); Yano \& Costa (1992, 2000); Yano \& Lisboa (1988); Yano \& Mello (1992); Yano \& Santos (1993); Yuzawa (1988, 1991) e Yuzawa \& Koike (1989).
Os padrões de distribuição foram baseados em dados da literatura sobre a distribuição geográfica de cada táxon.

Os estados brasileiros são apresentados por região geográfica e os nomes estão abreviados de acordo com o IBGE, como apresentado a seguir.

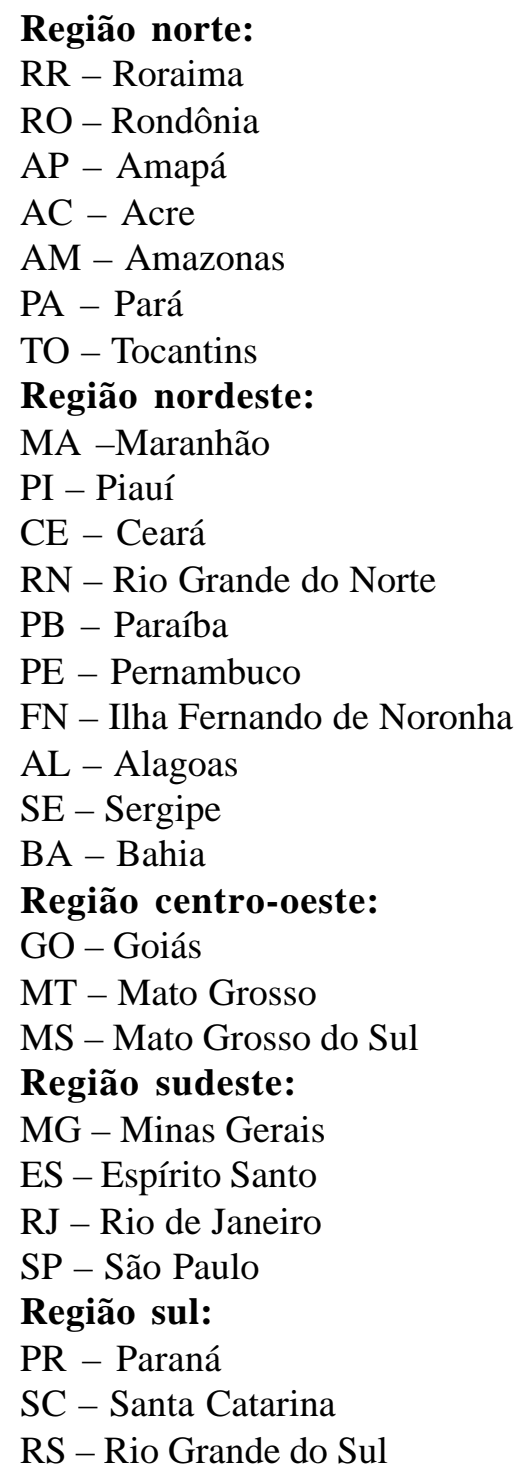

Todos os exemplares estão depositados na coleção de briófitas do herbário do Jardim Botânico do Rio de Janeiro (RB), com duplicatas para eventual intercâmbio. 


\section{RESULTADOS E DISCUSSÃO} Composição Florística

Foram encontradas no Arboreto do Jardim Botânico do Rio de Janeiro, 37 famílias de briófitas (1 de antóceros, 12 de hepáticas e 24 de musgos), em 61 gêneros (1 de antóceros, 23 de hepáticas e 37 de musgos), e 98 espécies (1 de antóceros, 40 de hepáticas e 57 de musgos), ocorrendo predominância de musgos (58\%) em relação as hepáticas (41\%) (Tabela 1). Este resultado é semelhante ao encontrado por Lisboa \& Ilkiu-Borges (1995), para Jardins Botânicos do município de Belém (PA), e por Costa (1999b), para formações primárias e secundárias de Mata Atlântica de Baixada no Estado do Rio de Janeiro.

A família Lejeuneaceae é a mais representativa dentre as hepáticas $(47,5 \%)$, com 11 gêneros e 19 espécies (Tabela 1), incluindo duas ocorrências novas para o Estado do Rio de Janeiro (Ceratolejeunea laetefusca (Austin) R. M. Schust. e Cololejeunea minutissima (Smith.) Schiffn. subsp. myriocarpa (Nees \& Mont.) R. M. Schust.). As famílias mais representativas de musgo são Bryaceae, Calymperaceae, Fissidentaceae e Sematophyllaceae (Tabela 1), as três primeiras com 5 espécies cada $(8,7 \%)$, e Sematophyllaceae com 6 espécies $(10,5 \%)$, enquanto o restante apresentou 4 ou menos espécies. Estas famílias estão entre as 15 principais encontradas em inventários florísticos de briófitas no Neotrópico (Gradstein \& Pócs, 1989).

Dentre as 98 espécies estudadas, 14 (14\%) foram encontradas nas copas das árvores, sendo que Acroporium longirostre (Brid.) W. R. Buck, Anoplolejeunea conferta (Meissn.) A. Evans e Syrrhopodon ligulatus Mont. estavam restritas, no parque, a este ambiente. Dessas três espécies, somente Anoplolejeunea conferta e Syrrhopodon ligulatus são citadas na literatura, como ocorrendo também nas copas de árvores (Gradstein, 1994 e Reese, 1993).

Por ser o arboreto uma área artificial e urbana, sofrendo influência direta da poluição, foram encontradas algumas espécies típicas de áreas perturbadas, como Barbula agraria Hedw., Bryum argentum Hedw., Frullania ericoides (Nees) Nees e Hyophila involuta (Hook.) A. Jaeger (Lisboa \& Ilkiu-Borges, 1995; Oliveira, 2001; Sergio, 1981).

Erpodium glaziovii Hampe geralmente é encontrado junto com Fabronia ciliaris (Brid.) Brid. var. polycarpa (Hook.) W. R. Buck e Frullania neesii Lindenb. Vital (1980), também observou a associação destas três espécies, comentando ainda, que são espécies comuns em parques e jardins.

Bryum renauldii Röl ex Ren. \& Card. é uma espécie cuja sexualidade era desconhecida (Sharp et al., 1994), e que no arboreto apresenta populações femininas com ramos caducos como forma de reprodução vegetativa. Observações feitas ao longo de 12 meses nunca revelaram a fase esporofítica ou mesmo populações masculinas. Segundo Oliveira (2001), este fato é observado em poucas espécies de musgos, existindo duas possibilidades em relação a sexualidade destes, ou a espécie é dióica e as populações masculinas são desconhecidas na natureza, ou é monóica e as condições ambientais não permitem a expressão do caráter masculino.

\section{Formas de Vida}

Foram caracterizados seis tipos de formas de vida (Tabela 1), predominando trama (32 espécies - 33\%), tufo (32 espécies - 33\%) e tapete (19 espécies - 19\%), totalizando 85\% das espécies estudadas (Gráfico 1). Este resultado é semelhante aos encontrados por Costa (1999b) e Montfoort \& Ek (1990) para as Florestas Tropicais de Terras Baixas, onde formas de vida agregadas como tapete, trama e tufo, são características e predominam em áreas abertas, com intensidade luminosa e umidade do ar altas.

Apesar de trama, tufo e tapete serem predominantes no parque, as talosas se destacam também devido ao grande número de espécies encontradas: Dumortiera hirsuta (Sw.) Nees, Marchantia chenopoda L., 
Metzgeria furcata (L.) Corda, $M$. psilocraspeda Schiffn., Monoclea gottschei Lindb. subsp. elongata Gradst. \& Mues, Phaeoceros laevis (L.) Prosk., Riccia curtisii (James ex Austin) Austin, $R$. stenophylla Spruce, Symphyogyna aspera Steph. e S. podophylla (Thumb.) Mont. \& Nees. Alguns desses táxons, como as espécies de Riccia, são xerotolerantes, estando adaptados a sobreviver em ambientes relativamente secos (Gradstein et al., 2001).

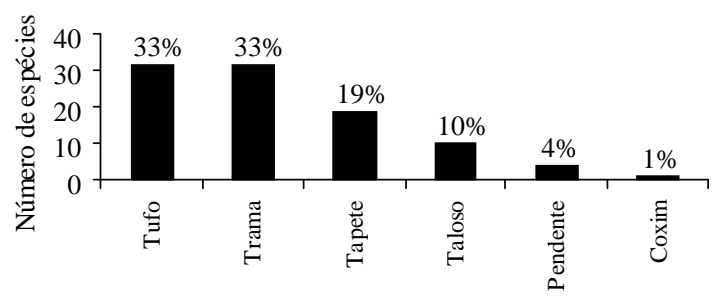

Grafico 1. Formas de vida das briófitas do arboreto do Jardim Botânico do Rio de Janeiro.

\section{Tipos de Substrato}

No arboreto do Jardim Botânico as briófitas colonizam diversos tipos de substrato: corticícola, epíxila, epífila, rupícola, terrícola e artificiais (Tabela 1). Dentre as 98 espécies, $39(40 \%)$ não apresentaram preferência por substrato, crescendo sobre mais de um tipo (Gráfico 2). Em relação às espécies que ocorreram em apenas um tipo de substrato, predominaram as corticícolas (35 espécies $36 \%$ ), o que era esperado por se tratar de um arboreto formado principalmente por árvores e arbustos. Nenhuma espécie estudada é exclusivamente epíxila, apesar deste ser um dos tipos de substrato colonizado.

Esta ausência de preferência por um tipo de substrato se deve, provavelmente, a uniformidade da estrutura de uma área artificial como a do arboreto do Jardim Botânico, com condições de luminosidade, temperatura e umidade do ar uniformes e ausência de um gradiente microclimático. Como exemplo, podemos citar Lejeunea flava (Sw.) Nees, que foi observada em quase todos os tipos de substratos, com exceção das folhas (epífila).
Algumas espécies ocorrem em substratos artificiais como o cimento da margem de canaletas, rios e canteiros do parque (Bryum argentum Hedw., Entodontopsis leucostega (Brid.) W. R. Buck \& Ireland, Fissidens palmatus Hedw., F. zollingeri Mont., Hyophyla involuta (Hook.) A. Jaeger, Lejeunea flava (Sw.) Nees, L. laetevirens Mont. \& Nees, Lophocolea martiana Nees e Monoclea gottschei Lindb. subsp. elongata Gradst. \& Mues); parede de pedra do bebedouro (Lejeunea laetevirens (Hedw.) A. Jaeger); portal de pedra da antiga Academia de Belas Artes (Helicodontium capillare (Hedw.) A. Jaeger); saco de areia dentro do Rio dos Macacos (Marchantia chenopoda L. e Riccia stenophylla Spruce). Outras como, Cololejeunea minutissima (Smith) Schiffn. subsp. myriocarpa (Nees \& Mont.) R. M. Schust., Lejeunea glaucescens Gottsche e Sematophyllum subpinnatum (Brid.) E. Britton, crescem sobre bambu (Tabela 1).

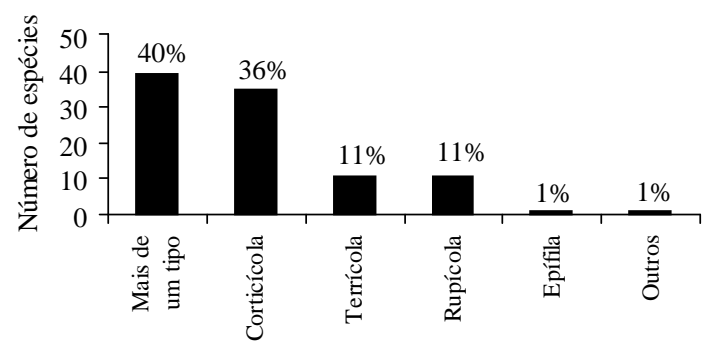

Grafico 2. Tipos de substrato colonizados no arboreto do Jardim Botânico do Rio de Janeiro.

\section{Distribuição Geográfica}

Dentre os padrões de distribuição caracterizados (Tabela 1 e Gráfico 3), predominou o Neotropical com 44 espécies (46\%), seguido do Pantropical com 15 espécies (16\%), Neotrópico e África com 9 espécies (10\%) e Cosmopolita com 9 espécies (10\%). Logo, $82 \%$ das espécies de briófitas do arboreto apresentam uma distribuição relativamente ampla no mundo, sendo este também um resultado esperado, visto que as briófitas, de uma maneira geral estão amplamente distribuídas.

Schiffneriolejeunea polycarpa (Nees) Gradst. é uma espécie pantropical, ocorrendo 
em florestas secundárias de baixa altitude, apresentando distribuição disjunta, entre o México e norte da América do Sul e o Sudeste do Brasil, estando ausente na região equatorial (Gradstein, 1994).

Bryum pseudocapillare Besch., Calymperes tenerum Müll. Hal., Ceratolejeunea laetefusca (Austin) R. M. Schust., Cololejeunea minutissima (Smith) Schiffn. subsp. myriocarpa (Nees \& Mont.) R. M. Schust. e Fissidens submarginatus Bruch são citados pela primeira vez para o estado do Rio de Janeiro. Bryum pseudocapillare (Bastos \& Bôas-Bastos, 1998), Bryum renauldii Röl ex Ren. \& Card. (Oliveira e Silva, 1998) e Calymperes tenerum Müll. Hal. (Visnadi, 1998), são citados pela segunda vez para o Brasil.

Leptophascum leptophyllum (Müll. Hal.) J. Guerra \& M. J. Cano foi citada por Oliveira e Silva (1998), como primeira referência para o Brasil, embora Sharp et al. (1994), já reportava a espécie para o Brasil sem citar a localidade.

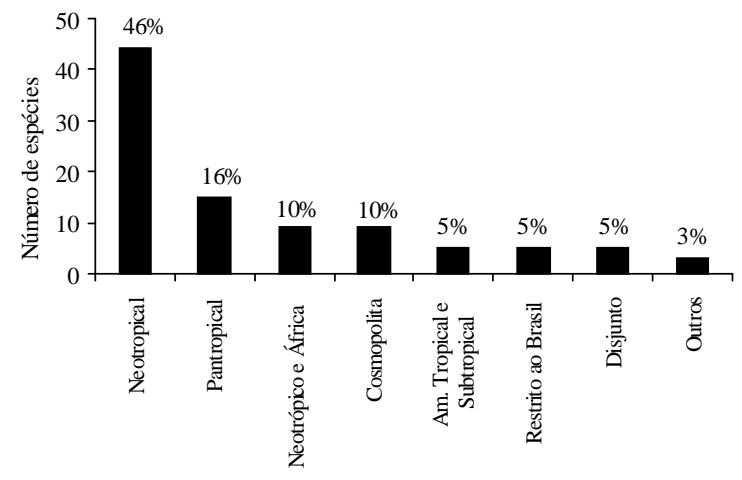

Gráfico 3. Padrões de distribuição geográfica das briófitas do arboreto do Jardim Botânico do Rio de Janeiro.

\section{Riqueza Florística}

Os resultados encontrados foram comparados com aqueles obtidos por Lisboa \& Ilkiu-Borges (1995), para Jardins Botânicos do município de Belém (PA) e por Costa (1999b), para a Reserva Biológica de Poço das Antas (RJ), por se tratar de uma área de Mata Atlântica de Baixada no Rio de Janeiro, com formações primárias e secundárias.

Apenas 16 (20\%) de um total de 80 táxons encontrados nos Jardins Botânicos do município de Belém (Tabela 2), ocorrem no parque (Barbula agraria Hedw., Callicostella pallida (Hornsch.) Aongstr., C. merkelii (Hornsch.) A. Jaeger, Calymperes lonchophyllum Schwaegr., C. palisotii Schwaegr. subsp. richardii (Müll. Hal.) S. Edwards, Fissidens guianensis Mont., Hyophila involuta (Hook.) A. Jaeger, Lejeunea flava (Sw.) Nees, L. glaucescens Gottsche, Leucobryum martianum (Hornsch.) Hampe, Lophocolea martiana Nees, Octoblepharum albidum Hedw., Philonotis uncinata (Schwaegr.) Brid., Sematophyllum adnatum (Michx.) E. Britton, S. subpinnatum (Brid.) E. Britton e Vesicularia vesicularis (Schwaegr.) Broth.).

Em relação a Poço das Antas (Tabela 2), de um total de 75 espécies, apenas 16 $(21,3 \%)$ são encontrados no arboreto (Callicostella merkelii (Horsnch.) A. Jaeger, Calymperes tenerum Müll. Hal., Frullania neesii Lindenb., Helicodontium capillare (Hedw.) A. Jaeger, Isopterygium tenerifolium Mitt., I. tenerum (Sw.) Mitt., Lejeunea flava (Sw.) Nees, L. glaucescens Gottsche, Leucobryum martianum (Hornsch.) Hampe, Leucolejeunea unciloba (Lindenb.) A. Evans, L. xanthocarpa (Lehm. \& Lindenb.) A. Evans, Lophocolea martiana Nees, Octoblepharum albidum Hedw., Pterogonidium pulchellum (Hook.) Müll. Hal., Sematophyllum subpinnatum (Brid.) E. Britton e Syrrhopodon incompletus Schwaegr.).

A diferença observada entre a brioflora destas três áreas não foi importante em relação ao número total de espécies e sim nos táxons encontrados. Este resultado comprova, em parte, a diferença observada entre a brioflora de áreas com florestas primárias e áreas com florestas secundárias (como áreas degradadas ou, plantadas ou urbanas), que sofrem influência direta da poluição, temperatura e luminosidade altas. Sergio (1981), estudando as alterações da brioflora na área urbana de Lisboa nos últimos 140 anos, encontrou resultados semelhantes. 
Dentre as 17 espécies do parque coletadas por Maria do Carmo Vaughan Bandeira, entre 1923-1927, e depositadas no herbário do Jardim Botânico, apenas Frullania dusenii Steph. e Squamidium brasiliense (Hornsch.) Broth. não foram reencontradas na área do arboreto. Isto se deve, provavelmente, à mudança das condições ambientais ao longo desses anos, principalmente o aumento da poluição do ar, visto que algumas espécies de briófitas são reconhecidamente sensíveis a este tipo de poluição (Rao, 1982).

Tabela 1. Brioflora do arboreto do Jardim Botânico. Forma de vida $(\mathrm{CX}=$ coxim, $\mathrm{P}=$ pendente, $\mathrm{TF}=$ tufo, $\mathrm{TL}=$ taloso, $\mathrm{TP}=$ tapete, $\mathrm{TR}=$ trama $)$. Tipo de Substrato $(\mathrm{C}=$ corticícola, $\mathrm{EX}=$ epíxila, $\mathrm{EF}=$ epífila, $\mathrm{O}=$ outros, $\mathrm{R}=$ rupícola, $\mathrm{T}=$ terrícola). Negrito $=$ ocorrência nova para o Estado do Rio de Janeiro. ${ }^{*}=$ Espécies do parque que estavam representadas no herbário antes deste estudo.

\begin{tabular}{|c|c|c|c|c|}
\hline Espécies & $\begin{array}{l}\text { Forma de } \\
\text { vida }\end{array}$ & $\begin{array}{l}\text { Tipo de } \\
\text { Substrato }\end{array}$ & $\begin{array}{c}\text { Variação } \\
\text { Altitudinal no } \\
\text { Brasil } \\
\end{array}$ & $\begin{array}{l}\text { Distribuição Geográfica } \\
\text { no mundo e no Brasil }\end{array}$ \\
\hline \multicolumn{5}{|l|}{ ANTHOCEROTOPHYTA (1) } \\
\hline \multicolumn{5}{|l|}{ ANTHOCEROTACEAE } \\
\hline Phaeoceros laevis (L.) Prosk.* & $\mathrm{TL}$ & $\mathrm{R}, \mathrm{T}$ & $0-1250 \mathrm{~m}$ & $\begin{array}{l}\text { Ampla. Brasil: AM, PE, } \\
\text { BA, MG, ES, RJ, SP, SC } \\
\text { e RS. }\end{array}$ \\
\hline \multicolumn{5}{|l|}{ НЕРАТОРНУТА (40) } \\
\hline \multicolumn{5}{|l|}{ CHONECOLACEAE } \\
\hline Chonecolea doellingeri (Nees) Grolle* & $\mathrm{TP}$ & $\mathrm{C}$ & $0-1000 \mathrm{~m}$ & $\begin{array}{l}\text { Pantropical. Brasil: MG, } \\
\text { ES, RJ, SP, e RS. }\end{array}$ \\
\hline \multicolumn{5}{|l|}{ CYLiNDROCOLEACEAE } \\
\hline $\begin{array}{l}\text { Cylindrocolea rhizantha (Mont.) R. M. } \\
\text { Schust. }\end{array}$ & $\mathrm{TP}$ & $\mathrm{C}$ & $0-1000 \mathrm{~m}$ & $\begin{array}{l}\text { Neotropical. Brasil: PE, } \\
\text { BA, ES, RJ e SP. }\end{array}$ \\
\hline \multicolumn{5}{|l|}{ FOSSOMBRONIACEAE } \\
\hline $\begin{array}{l}\text { Fossombronia porphyrorhiza (Nees) } \\
\text { Prosk. }\end{array}$ & $\mathrm{TR}$ & $\mathrm{R}$ & $0-1100 \mathrm{~m}$ & $\begin{array}{l}\text { Neotropical. Brasil: PE, } \\
\text { MT, MG, ES, RJ e SP. }\end{array}$ \\
\hline \multicolumn{5}{|l|}{ FRULLANIACEAE } \\
\hline Frullania caulisequa (Nees) Nees* & $\mathrm{TP}$ & $\mathrm{C}$ & $0-1000 \mathrm{~m}$ & $\begin{array}{l}\text { Neotropical. Brasil: RR, } \\
\text { AC, PA, PE, SE, BA, } \\
\text { MG, ES, RJ, SP, SC e } \\
\text { RS. }\end{array}$ \\
\hline F. ericoides (Nees) Nees $*$ & $\mathrm{TP}$ & $\mathrm{C}, \mathrm{R}$ & $0-1300 \mathrm{~m}$ & $\begin{array}{l}\text { Pantropical. Brasil: PA, } \\
\text { PB, PE, FN, BA, GO, } \\
\text { MT, MG, ES, RJ, SP e } \\
\text { RS. }\end{array}$ \\
\hline F. neesii Lindenb.* & $\mathrm{TP}$ & $\mathrm{C}$ & $0-2400 \mathrm{~m}$ & $\begin{array}{l}\text { Neotropical. Brasil: RR, } \\
\text { AC, AM, CE, PB, PE, } \\
\text { SE, BA, MT, MG, ES, } \\
\text { RJ, SP, PR e RS. }\end{array}$ \\
\hline F. riojaneirensis (Raddi) Aongstr. & $\mathrm{TP}$ & $\mathrm{C}$ & $0-1100 \mathrm{~m}$ & $\begin{array}{l}\text { Pantropical. Brasil: PA, } \\
\text { PB, PE, BA, DF, GO, } \\
\text { MT, MG, ES, RJ, SP e } \\
\text { RS. }\end{array}$ \\
\hline \multicolumn{5}{|l|}{ LEJEUNEACEAE } \\
\hline $\begin{array}{l}\text { Anoplolejeunea conferta (Meissn.) A. } \\
\text { Evans }\end{array}$ & TR & $\mathrm{C}$ & $0-2400 \mathrm{~m}$ & $\begin{array}{l}\text { Neotropical. Brasil: PE, } \\
\text { BA. MG, ES, RJ, SP e } \\
\text { RS. }\end{array}$ \\
\hline Archilejeunea parviflora (Nees) Schiffn. & TR & $\mathrm{C}, \mathrm{R}$ & $0-1500 \mathrm{~m}$ & $\begin{array}{l}\text { Neotropical. Brasil: RR, } \\
\text { RO, AC, AM, PA, PE, } \\
\text { MG, ES, RJ e SP. }\end{array}$ \\
\hline $\begin{array}{l}\text { Ceratolejeunea laetefusca (Austin) R. M. } \\
\text { Schust. }\end{array}$ & TR & $\mathrm{C}$ & $0-1300 \mathrm{~m}$ & $\begin{array}{l}\text { Neotropical. Brasil: AC, } \\
\text { AM, PA, PE, MG, ES, } \\
\text { RJ e SP. }\end{array}$ \\
\hline $\begin{array}{l}\text { Cheilolejeunea rigidula (Mont.) R. M. } \\
\text { Schust. }\end{array}$ & $\mathrm{TP}$ & $\mathrm{C}$ & $0-1000 \mathrm{~m}$ & $\begin{array}{l}\text { Neotrópico e África } \\
\text { tropical. Brasil: AC, AM, } \\
\text { PE, BA, ES, RJ e SP. }\end{array}$ \\
\hline
\end{tabular}

Rodriguésia 52(81): 107-124. 2001 


\begin{tabular}{|c|c|c|c|c|}
\hline Espécies & $\begin{array}{l}\text { Forma de } \\
\text { vida }\end{array}$ & $\begin{array}{l}\text { Tipo de } \\
\text { Substrato }\end{array}$ & $\begin{array}{c}\text { Variação } \\
\text { Altitudinal no } \\
\text { Brasil } \\
\end{array}$ & $\begin{array}{c}\text { Distribuição Geográfica } \\
\text { no mundo e no Brasil }\end{array}$ \\
\hline $\begin{array}{l}\text { Cololejeunea cardiocarpa (Mont.) A. } \\
\text { Evans }\end{array}$ & TR & $\mathrm{EF}$ & $0-1000 \mathrm{~m}$ & $\begin{array}{l}\text { Pantropical. Brasil: RR, } \\
\text { AM, PB, ES, RJ, e SP. }\end{array}$ \\
\hline $\begin{array}{l}\text { C. minutissima (Smith.) Schiffn. subsp. } \\
\text { myriocarpa (Nees \& Mont.) R. M. Schust. }\end{array}$ & TR & $\mathrm{C}, \mathrm{O}$ & $0-1000 \mathrm{~m}$ & $\begin{array}{l}\text { Pantropical. Brasil: RJ e } \\
\text { SP. }\end{array}$ \\
\hline Drepanolejeunea mosenii (Steph.) Bischl. & TR & $\mathrm{C}$ & $0-2000 \mathrm{~m}$ & $\begin{array}{l}\text { Neotropical. Brasil: AM, } \\
\text { PE, MG, ES, RJ, SP, PR, } \\
\text { SC e RS. }\end{array}$ \\
\hline Lejeunea caespitosa Lindenb. & TR & $\mathrm{R}, \mathrm{C}$ & $0-800 \mathrm{~m}$ & $\begin{array}{l}\text { Neotrópico e África. } \\
\text { Brasil: AC, PA, RJ e SP. }\end{array}$ \\
\hline L. cristulata (Steph.) E. Reiner \& Goda & TR & $\mathrm{C}, \mathrm{R}$ & $0-1500 \mathrm{~m}$ & $\begin{array}{l}\text { Restrito ao Brasil.: PE, } \\
\text { MG, RJ, SP e SC. }\end{array}$ \\
\hline L flava (Sw.) Nees* & TR & $\mathrm{C}, \mathrm{R}, \mathrm{EX}, \mathrm{O}$ & $0-2400 \mathrm{~m}$ & $\begin{array}{l}\text { Pantropical. . Brasil: RR, } \\
\text { AC, AM, PA, PE, GO, } \\
\text { BA, MG, ES, RJ, SP e } \\
\text { RS. }\end{array}$ \\
\hline L. glaucescens Gottsche* & TR & $\mathrm{C}, \mathrm{R}, \mathrm{T}, \mathrm{O}$ & $0-1100 \mathrm{~m}$ & $\begin{array}{l}\text { Neotropical. Brasil: AC, } \\
\text { PA, PE, BA, ES, RJ e } \\
\text { SP. }\end{array}$ \\
\hline L. laetevirens Mont. \& Nees & TR & $\mathrm{C}, \mathrm{R}, \mathrm{T}, \mathrm{O}$ & $0-1500 \mathrm{~m}$ & $\begin{array}{l}\text { Neotropical. Brasil: PA, } \\
\text { PE, FN, BA, ES, RJ e } \\
\text { SP. }\end{array}$ \\
\hline L. phylobolla (A. Evans) Grolle & TR & $\mathrm{C}$ & $0-800 \mathrm{~m}$ & $\begin{array}{l}\text { Neotrópico e África. } \\
\text { Brasil: PA, ES, RJ e SP. }\end{array}$ \\
\hline L. trinitensis Lindenb. & TR & $\mathrm{C}$ & $0-800 \mathrm{~m}$ & $\begin{array}{l}\text { Disjunto, ocorrendo na } \\
\text { América tropical e África } \\
\text { (Comores). Brasil: AC, } \\
\text { AM, GO, MT, MS, BA, } \\
\text { MG, ES, RJ, SP e PR. }\end{array}$ \\
\hline $\begin{array}{l}\text { Leucolejeunea unciloba (Lindenb.) A. } \\
\text { Evans }\end{array}$ & TR & $\mathrm{C}$ & $0-1300 \mathrm{~m}$ & $\begin{array}{l}\text { Neotrópico e África. } \\
\text { Brasil: PE, BA, ES, RJ e } \\
\text { SP }\end{array}$ \\
\hline $\begin{array}{l}\text { L. xanthocarpa (Lehm. \& Lindenb.) A. } \\
\text { Evans }\end{array}$ & TR & $\mathrm{R}, \mathrm{C}$ & $0-2500 \mathrm{~m}$ & $\begin{array}{l}\text { Pantropical. Brasil: PE, } \\
\text { BA, MG, ES, RJ, SP e } \\
\text { SC. }\end{array}$ \\
\hline Microlejeunea bullata (Taylor) Steph. & TR & $\mathrm{C}$ & $0-2400 \mathrm{~m}$ & $\begin{array}{l}\text { Neotropical. Brasil: RR, } \\
\text { AC, SE, ES, RJ e SP. }\end{array}$ \\
\hline Omphalanthus filiformis (Sw.) Nees & TR & $\mathrm{C}$ & $0-2000 \mathrm{~m}$ & $\begin{array}{l}\text { Neotropical Brasil: AM, } \\
\text { PE, BA, MG, ES, RJ e } \\
\text { SP. }\end{array}$ \\
\hline $\begin{array}{l}\text { Schiffneriolejeunea polycarpa (Nees) } \\
\text { Gradst. }\end{array}$ & TR & $\mathrm{C}$ & $0-1000 \mathrm{~m}$ & $\begin{array}{l}\text { Pantropical. Brasil: AM, } \\
\text { PA, PE, BA, GO, MG, } \\
\text { ES, RJ, SP, SC e RS. }\end{array}$ \\
\hline \multicolumn{5}{|l|}{ LOPHOCOLEACEAE } \\
\hline Lophocolea bidentata (L.) Dumort. & TR & $\mathrm{R}$ & $0-1500 \mathrm{~m}$ & $\begin{array}{l}\text { Ampla. Brasil: AC, AM, } \\
\text { GO, MG, ES, RJ, SP e } \\
\text { RS. }\end{array}$ \\
\hline L. martiana Nees* & TR & $\mathrm{T}, \mathrm{R}, \mathrm{O}$ & $0-1850 \mathrm{~m}$ & $\begin{array}{l}\text { Neotrópico e África. } \\
\text { Brasil: AP, AM, PA, PE, } \\
\text { SE, BA, MG, ES, RJ, } \\
\text { SP, PR, SC e RS. }\end{array}$ \\
\hline \multicolumn{5}{|l|}{ MARCHANTIACEAE } \\
\hline Dumortiera hirsuta (Sw.) Nees & TL & $\mathrm{T}$ & $0-2000 \mathrm{~m}$ & $\begin{array}{l}\text { Ampla. Brasil: AC, AM, } \\
\text { PA, MT, DF, MG, ES, } \\
\text { RJ, SP, PR, SC e RS. }\end{array}$ \\
\hline Marchantia chenopoda $\mathrm{L}$. & $\mathrm{TL}$ & $\mathrm{R}, \mathrm{O}$ & $0-1500 \mathrm{~m}$ & $\begin{array}{l}\text { Neotropical Brasil: AM, } \\
\text { MT, DF, MG, ES, RJ, } \\
\text { SP, PR e RS. }\end{array}$ \\
\hline
\end{tabular}




\begin{tabular}{|c|c|c|c|c|}
\hline Espécies & $\begin{array}{l}\text { Forma de } \\
\text { vida }\end{array}$ & $\begin{array}{l}\text { Tipo de } \\
\text { Substrato }\end{array}$ & $\begin{array}{c}\text { Variação } \\
\text { Altitudinal no } \\
\text { Brasil }\end{array}$ & $\begin{array}{c}\text { Distribuição Geográfica } \\
\text { no mundo e no Brasil }\end{array}$ \\
\hline \multicolumn{5}{|l|}{ METZGERIACEAE } \\
\hline Metzgeria furcata (L.) Corda & $\mathrm{TL}$ & $\mathrm{C}$ & $0-1500 \mathrm{~m}$ & $\begin{array}{l}\text { Ampla. Brasil: AC, PE, } \\
\text { BA, GO, RJ, SP, PR e } \\
\text { RS. }\end{array}$ \\
\hline M. psilocraspeda Schiffn. & $\mathrm{TL}$ & $\mathrm{C}$ & $0-2000 \mathrm{~m}$ & $\begin{array}{l}\text { Restrito ao Brasil.: MG, } \\
\text { ES, RJ, SP, PR e SC. }\end{array}$ \\
\hline \multicolumn{5}{|l|}{ MONOCLEACEAE } \\
\hline $\begin{array}{l}\text { Monoclea gottschei Lindb. subsp. elongata } \\
\text { Gradst. \& Mues }\end{array}$ & $\mathrm{TL}$ & $\mathrm{R}, \mathrm{O}$ & $0-2000 \mathrm{~m}$ & $\begin{array}{l}\text { América tropical e } \\
\text { subtropical. Brasil: AM, } \\
\text { PE, RJ, SP e RS. }\end{array}$ \\
\hline \multicolumn{5}{|l|}{ PallaViciniaceae } \\
\hline Symphyogyna aspera Steph. & TL & $\mathrm{T}$ & $0-2200 \mathrm{~m}$ & $\begin{array}{l}\text { Neotropical. Brasil: AM, } \\
\text { PE, MG, ES, RJ, SP, SC } \\
\text { e RS. }\end{array}$ \\
\hline S. podophylla (Thumb.) Mont. \& Nees & $\mathrm{TL}$ & $\mathrm{T}$ & $0-1800 \mathrm{~m}$ & $\begin{array}{l}\text { Disjunto, ocorrendo no } \\
\text { neotrópico e regiões } \\
\text { temperadas } \\
\text { hemisfério Sul. Brasil: } \\
\text { AM, MG, ES, RJ e SP. }\end{array}$ \\
\hline \multicolumn{5}{|l|}{ Plagiochilaceae } \\
\hline $\begin{array}{l}\text { Plagiochila corrugata (Nees) Nees \& } \\
\text { Mont. }\end{array}$ & $P$ & $\mathrm{C}$ & $0-2300 \mathrm{~m}$ & $\begin{array}{l}\text { Neotropical. Brasil: } \\
\text { PE,BA, MG, ES, RJ, SP, } \\
\text { PR, SC e RS. }\end{array}$ \\
\hline$P$ martiana (Nees) Lindenb. & $\mathrm{P}$ & $\mathrm{C}, \mathrm{R}$ & $0-1100 \mathrm{~m}$ & $\begin{array}{l}\text { Neotropical. Brasil: PE, } \\
\text { MG, RJ, SP, SC e RS. }\end{array}$ \\
\hline P. raddiana Lindenb. & $\mathrm{P}$ & $\mathrm{C}$ & $0-1350 \mathrm{~m}$ & $\begin{array}{l}\text { Neotropical. Brasil: PA, } \\
\text { PE, MT, MG, ES, RJ, SP } \\
\text { e PR }\end{array}$ \\
\hline \multicolumn{5}{|l|}{ RICCIACEAE } \\
\hline $\begin{array}{l}\text { Riccia aff. curtisii (James ex. Austin) } \\
\text { Austin }\end{array}$ & $\mathrm{TL}$ & $\mathrm{T}$ & $0-500 \mathrm{~m}$ & $\begin{array}{l}\text { América tropical e } \\
\text { subtropical. Brasil: ES, } \\
\text { RJ, SP e RS. }\end{array}$ \\
\hline R. stenophylla Spruce & $\mathrm{TL}$ & $\mathrm{R}, \mathrm{T}, \mathrm{O}$ & $0-1000 \mathrm{~m}$ & $\begin{array}{l}\text { América tropical e } \\
\text { subtropical. Brasil: PE, } \\
\text { BA, GO, MT, ES, RJ, } \\
\text { SP, PR, SC e RS. }\end{array}$ \\
\hline \multicolumn{5}{|l|}{ BRYOPHYTA (57) } \\
\hline \multicolumn{5}{|l|}{ BARTRAMIACEAE } \\
\hline Philonotis gardneri (Müll. Hal.) A. Jaeger & $\mathrm{TF}$ & $\mathrm{T}, \mathrm{C}$ & $0-2000 \mathrm{~m}$ & $\begin{array}{l}\text { Restrito ao Brasil: MG, } \\
\text { RJ, SP, PR e RS. }\end{array}$ \\
\hline P. uncinata (Schwaegr.) Brid. & $\mathrm{TF}$ & $\mathrm{R}$ & $0-800 \mathrm{~m}$ & $\begin{array}{l}\text { Pantropical. Brasil: RO, } \\
\text { AM, PA, PI, PB, PE, } \\
\text { BA, GO, MT, MG, ES, } \\
\text { RJ e sc. }\end{array}$ \\
\hline \multicolumn{5}{|l|}{ BRACHYTHECIACEAE } \\
\hline Brachythecium sp. & TR & $\mathrm{C}$ & & \\
\hline $\begin{array}{l}\text { Rhynchostegium scariosum (Taylor) A. } \\
\text { Jaeger }\end{array}$ & $\mathrm{TP}$ & $\mathrm{T}$ & $0-800 \mathrm{~m}$ & $\begin{array}{l}\text { Neotropical. Brasil: PE, } \\
\text { RJ, SP e RS. }\end{array}$ \\
\hline \multicolumn{5}{|l|}{ BRUCHIACEAE } \\
\hline Trematodon longicollis Michx. & $\mathrm{TP}$ & $\mathrm{T}$ & $0-800 \mathrm{~m}$ & $\begin{array}{l}\text { Neotrópico, Ásia, Havaí } \\
\text { e Papua - Nova Guiné. } \\
\text { Brasil: RO, PA, PE, ES, } \\
\text { RJ, SP, PR, SC e RS. }\end{array}$ \\
\hline \multicolumn{5}{|l|}{ BRYACEAE } \\
\hline Bryum argenteum Hedw. & TF & $\mathrm{O}$ & $0-1300 \mathrm{~m}$ & $\begin{array}{l}\text { Ampla. Brasil: AM, CE, } \\
\text { PB, PE, AL BA, DF, } \\
\text { GO, MT, MG, ES, RJ, } \\
\text { SP, PR, SC e RS. }\end{array}$ \\
\hline
\end{tabular}




\begin{tabular}{|c|c|c|c|c|}
\hline Espécies & $\begin{array}{l}\text { Forma de } \\
\text { vida }\end{array}$ & $\begin{array}{l}\text { Tipo de } \\
\text { Substrato }\end{array}$ & $\begin{array}{c}\text { Variação } \\
\text { Altitudinal no } \\
\text { Brasil } \\
\end{array}$ & $\begin{array}{l}\text { Distribuição Geográfica } \\
\text { no mundo e no Brasil }\end{array}$ \\
\hline B. densifolium Brid. & $\mathrm{TF}$ & $\mathrm{T}, \mathrm{R}, \mathrm{EX}, \mathrm{C}$ & $0-1200 \mathrm{~m}$ & $\begin{array}{l}\text { Neotropical. Brasil: AC, } \\
\text { PE, BA, DF, MG, ES, } \\
\text { RJ, SP, PR, SC e RS. }\end{array}$ \\
\hline B. limbatum Müll. Hal. & $\mathrm{TF}$ & R, EX & $0-800 \mathrm{~m}$ & $\begin{array}{l}\text { Neotropical. Brasil: MG, } \\
\text { ES, RJ, SP, PR, SC e RS. }\end{array}$ \\
\hline Bryum pseudocapillare Besch. & $\mathrm{TF}$ & $\mathrm{C}$ & nível do mar & $\begin{array}{l}\text { América tropical e } \\
\text { subtropical. Brasil: BA e } \\
\text { RJ. }\end{array}$ \\
\hline $\begin{array}{l}\text { B. renauldii Röl ex. Ren. \& Card. } \\
\text { CALLICOSTACEAE }\end{array}$ & $\mathrm{TF}$ & $\mathrm{R}$ & nível do mar & Neotropical. Brasil: RJ. \\
\hline Calliscotella merkelli (Hornsch.) A. Jaeger & TR & $\mathrm{T}, \mathrm{R}, \mathrm{EX}$ & $0-800 \mathrm{~m}$ & $\begin{array}{l}\text { Neotropical. Brasil: PA, } \\
\text { MG, RJ, SP e SC. }\end{array}$ \\
\hline C. pallida (Hornsch.) Aongstr. & TR & $\mathrm{R}$ & $0-1300 \mathrm{~m}$ & $\begin{array}{l}\text { Neotropical. Brasil: RR, } \\
\text { RO, AP, AC, AM, PA, } \\
\text { PE, SE, BA, GO, MT, } \\
\text { MG, ES, RJ, SP, PR e } \\
\text { RS. }\end{array}$ \\
\hline \multicolumn{5}{|l|}{ CALYMPERACEAE } \\
\hline Calymperes lonchophyllum Schwaegr. & $\mathrm{TF}$ & $\mathrm{R}$ & $0-800 \mathrm{~m}$ & $\begin{array}{l}\text { Pantropical. Brasil: RR, } \\
\text { RO, AP, AC, AM, PA, } \\
\text { MA, PE, AL, BA, MT, } \\
\text { ES, RJ, SP e PR. }\end{array}$ \\
\hline $\begin{array}{l}\text { C. palisotii Schwaegr. subsp. richardii } \\
\text { (Müll. Hal.) S. Edwards }\end{array}$ & $\mathrm{TF}$ & $\mathrm{C}, \mathrm{R}$ & $0-200 \mathrm{~m}$ & $\begin{array}{l}\text { Neotrópico, África } \\
\text { tropical e oeste da } \\
\text { Ásia.Brasil: RO, AP, } \\
\text { AM, PA, RN, PB, PE, } \\
\text { FN, AL, BA, go, es, rj e } \\
\text { pr. }\end{array}$ \\
\hline C. tenerum Müll. Hal. & $\mathrm{TF}$ & $\mathrm{C}, \mathrm{R}$ & nível do mar & $\begin{array}{l}\text { Pantropical.Brasil: } \mathrm{RJ} \text { e } \\
\text { SP. }\end{array}$ \\
\hline Syrrhopodon incompletus Schwaegr. & $\mathrm{TF}$ & $\mathrm{C}$ & $0-800 \mathrm{~m}$. & $\begin{array}{l}\text { América tropical e } \\
\text { subtropical e África } \\
\text { tropical. Brasil: RR, RO, } \\
\text { AP, AC, AM, PA, PE, } \\
\text { BA, GO, MT, RJ, SP e } \\
\text { SC. }\end{array}$ \\
\hline S. ligulatus Mont. & $\mathrm{TF}$ & $\mathrm{C}$ & $0-800 \mathrm{~m}$ & $\begin{array}{l}\text { Neotropical. Brasil: RR, } \\
\text { RO, AP, AM, PA, PE, } \\
\text { BA, GO, MT, MG, RJ e } \\
\text { SP. }\end{array}$ \\
\hline \multicolumn{5}{|l|}{ DICRANACEAE } \\
\hline Campylopus dichrostis (Müll. Hal.) Paris & $\mathrm{TF}$ & $\mathrm{C}$ & $0-800 \mathrm{~m}$. & $\begin{array}{l}\text { Restrito ao Brasil: BA, } \\
\text { GO, MG e RJ. }\end{array}$ \\
\hline C. julicaulis Broth. & $\mathrm{TF}$ & $\mathrm{C}$ & $0-2200 \mathrm{~m}$ & $\begin{array}{l}\text { Restrito ao Brasil: BA, } \\
\text { RJ, SP, PR, SC e RS. }\end{array}$ \\
\hline C. occultus Mitt. & $\mathrm{TF}$ & $\mathrm{C}$ & $0-2500 \mathrm{~m}$ & $\begin{array}{l}\text { Sul da América do sul. } \\
\text { Brasil: MA, PE, BA, } \\
\text { GO, MG, ES, RJ, SP, PR } \\
\text { e RS. }\end{array}$ \\
\hline \multicolumn{5}{|l|}{ ENTODONTACEAE } \\
\hline Erythrodontium longisetum (Hook.) Paris & $\mathrm{TF}$ & $\mathrm{C}, \mathrm{O}$ & $0-800 \mathrm{~m}$ & $\begin{array}{l}\text { Neotropical. Brasil: PE, } \\
\text { GO, MG, RJ, SP, PR e } \\
\text { RS. }\end{array}$ \\
\hline
\end{tabular}




\begin{tabular}{|c|c|c|c|c|}
\hline Espécies & $\begin{array}{l}\text { Forma de } \\
\text { vida }\end{array}$ & $\begin{array}{l}\text { Tipo de } \\
\text { Substrato }\end{array}$ & $\begin{array}{c}\text { Variação } \\
\text { Altitudinal no } \\
\text { Brasil } \\
\end{array}$ & $\begin{array}{c}\text { Distribuição Geográfica } \\
\text { no mundo e no Brasil }\end{array}$ \\
\hline \multicolumn{5}{|l|}{ ERPODIACEAE } \\
\hline Erpodium glaziovii Hampe* & $\mathrm{TF}$ & $\mathrm{C}$ & $0-800 \mathrm{~m}$ & $\begin{array}{l}\text { Neotropical. Brasil: AM, } \\
\text { BA, MS, MG, ES, RJ, } \\
\text { SP, PR, SC e RS. }\end{array}$ \\
\hline \multicolumn{5}{|l|}{ FABRONIACEAE } \\
\hline $\begin{array}{l}\text { Fabronia ciliaris (Brid.) Brid. var. } \\
\text { polycarpa }(\text { Hook.) W. R. Buck }\end{array}$ & $\mathrm{TP}$ & $\mathrm{C}$ & $0-800 \mathrm{~m}$ & $\begin{array}{l}\text { Neotropical. Brasil: CE, } \\
\text { PB, PE, SE, BA, GO, } \\
\text { MG, ES, RJ, SP, PR, SC } \\
\text { e RS. }\end{array}$ \\
\hline \multicolumn{5}{|l|}{ FISSIDENTACEAE } \\
\hline Fissidens guianensis Mont. & $\mathrm{TF}$ & $\mathrm{R}, \mathrm{EX}, \mathrm{T}$ & $0-500 \mathrm{~m}$ & $\begin{array}{l}\text { Neotropical. Brasil: RO, } \\
\text { AM, PA, PI, PE, GO, } \\
\text { MT, RJ, SP e SC. }\end{array}$ \\
\hline Fissidens palmatus Hedw. & $\mathrm{TF}$ & $\mathrm{T}, \mathrm{R}, \mathrm{O}$ & $0-800 \mathrm{~m}$ & $\begin{array}{l}\text { Neotropical. Brasil: RO, } \\
\text { CE, PE, FN, MG e RJ. }\end{array}$ \\
\hline F. radicans Mont. & $\mathrm{TF}$ & $\mathrm{T}, \mathrm{R}$ & $0-200 \mathrm{~m}$ & $\begin{array}{l}\text { Neotropical. Brasil: PA, } \\
\text { PB, PE, BA, RJ, SP, PR } \\
\text { e RS. }\end{array}$ \\
\hline F. submarginatus Bruch & $\mathrm{TF}$ & $\mathrm{C}, \mathrm{T}$ & $0-500 \mathrm{~m}$ & $\begin{array}{l}\text { Neotropical. Brasil: RO, } \\
\text { AM, PE, FN e RJ. }\end{array}$ \\
\hline F. zollingeri Mont. & $\mathrm{TF}$ & $\mathrm{T}, \mathrm{R}, \mathrm{O}$ & $0-800 \mathrm{~m}$ & $\begin{array}{l}\text { Ampla. Brasil: RO AC, } \\
\text { AM, PA, TO, MA PI, } \\
\text { PB, PE, FN, BA, GO, } \\
\text { MS, MG, ES, RJ SP, PR } \\
\text { e SC. }\end{array}$ \\
\hline \multicolumn{5}{|l|}{ FUNARIACEAE } \\
\hline Physcomitrium sp. & TF & $\mathrm{T}$ & & \\
\hline $\begin{array}{l}\text { HYPNACEAE } \\
\text { Isopterigium tenerifolium Mitt. }\end{array}$ & $\mathrm{TP}$ & $\mathrm{C}, \mathrm{EX}$ & $0-2000 \mathrm{~m}$ & $\begin{array}{l}\text { América tropical e } \\
\text { subtropical. Brasil: AM, } \\
\text { PA, CE, BA, GO, MG, } \\
\text { RJ, SP, PR, SC e RS. }\end{array}$ \\
\hline I. tenerum (Sw.) Mitt.* & $\mathrm{TP}$ & $\mathrm{C}, \mathrm{R}, \mathrm{EX}$ & $0-1200 \mathrm{~m}$ & $\begin{array}{l}\text { Disjunto, ocorrendo no } \\
\text { Neotrópico, oeste da } \\
\text { América do Norte e } \\
\text { Europa. Brasil: RR, AC, } \\
\text { AM, PA, PI, PB, PE, } \\
\text { BA, GO, MT, MG, ES, } \\
\text { RJ, SP, PR, SC e RS. }\end{array}$ \\
\hline $\begin{array}{l}\text { Vesicularia vesicularis } \quad \text { (Schwaegr.) } \\
\text { Broth.* }\end{array}$ & $\mathrm{TP}$ & $\mathrm{EX}, \mathrm{C}, \mathrm{T}, \mathrm{R}$ & $0-800 \mathrm{~m}$ & $\begin{array}{l}\text { Neotropical (alcançando } \\
\text { os SE EUA). Brasil: AM, } \\
\text { PA, pi, BA, MG, ES, RJ, } \\
\text { SP, PR, SC e RS. }\end{array}$ \\
\hline $\begin{array}{l}\text { LESKEACEAE } \\
\text { Hapocladium microphyllum (Hedw.) } \\
\text { Broth. }\end{array}$ & $\mathrm{TP}$ & $\mathrm{T}$ & $0-800 \mathrm{~m}$ & $\begin{array}{l}\text { Disjunto, ocorrendo na } \\
\text { América tropical e } \\
\text { subtropical e oeste da } \\
\text { Ásia. Brasil: DF, GO, } \\
\text { MG, RJ, SP PR, SC e } \\
\text { RS. }\end{array}$ \\
\hline $\begin{array}{l}\text { LEUCOBRYACEAE } \\
\text { Leucobryum martianum (Hornsch.) Hampe }\end{array}$ & $\mathrm{CX}$ & $\mathrm{T}$ & $0-800 \mathrm{~m}$ & $\begin{array}{l}\text { Neotropical. Brasil: RR, } \\
\text { RO, AP, AC, AM, PA, } \\
\text { MA, CE, PE, SE, BA, } \\
\text { MT, MG, ES, RJ, SP, PR } \\
\text { e SC. }\end{array}$ \\
\hline
\end{tabular}




\begin{tabular}{|c|c|c|c|c|}
\hline Espécies & $\begin{array}{l}\text { Forma de } \\
\text { vida }\end{array}$ & $\begin{array}{l}\text { Tipo de } \\
\text { Substrato }\end{array}$ & $\begin{array}{c}\text { Variação } \\
\text { Altitudinal no } \\
\text { Brasil } \\
\end{array}$ & $\begin{array}{c}\text { Distribuição Geográfica } \\
\text { no mundo e no Brasil }\end{array}$ \\
\hline Octoblepharum albidum Hedw.* & $\mathrm{TF}$ & $\mathrm{C}, \mathrm{EX}$ & $0-800 \mathrm{~m}$ & $\begin{array}{l}\text { Pantropical (alcançando } \\
\text { o SE do EUA). Brasil: } \\
\text { RR, RO, AP, AC, AM, } \\
\text { PA, TO, MA, CE, PB, } \\
\text { PE, FN, SE, BA, GO, } \\
\text { MT, MG, ES, RJ, SP, PR } \\
\text { e SC. }\end{array}$ \\
\hline \multicolumn{5}{|l|}{ METEORIACEAE } \\
\hline $\begin{array}{l}\text { Meteorium nigrescens (Hedw.) Dozy \& } \\
\text { Molk. }\end{array}$ & $\mathrm{P}$ & $\mathrm{C}, \mathrm{R}$ & $0-2000 \mathrm{~m}$ & $\begin{array}{l}\text { Pantropical. Brasil: PE, } \\
\text { BA, MG, ES, RJ, SP, } \\
\text { PR, SC e RS. }\end{array}$ \\
\hline Zelometeorium patulum (Hedw.) Manuel & $\mathrm{TP}$ & $\mathrm{R}$ & $0-1400 \mathrm{~m}$ & $\begin{array}{l}\text { Neotropical (alcançando } \\
\text { a Argentina). Brasil: } \\
\text { AM, AL, GO, MT, MG, } \\
\text { ES, RJ, SP, PR, SC e RS. }\end{array}$ \\
\hline $\begin{array}{l}\text { MYRINIACEAE } \\
\text { Helicodontium capillare (Hedw.) A. } \\
\text { Jaeger* }\end{array}$ & TR & $\mathrm{R}, \mathrm{C}, \mathrm{O}$ & $0-800 \mathrm{~m}$ & $\begin{array}{l}\text { Neotropical. Brasil: AC, } \\
\text { BA, GO, MG, ES, RJ, } \\
\text { SP, PR, SC e RS. }\end{array}$ \\
\hline \multicolumn{5}{|l|}{ ORTHOTRICHACEAE } \\
\hline Macromitrium cirrosum (Hedw.) Brid. & $\mathrm{TF}$ & $\mathrm{C}$ & $0-800 \mathrm{~m}$ & $\begin{array}{l}\text { Neotropical. Brasil: PA, } \\
\text { BA, RJ, SP, PR e SC. }\end{array}$ \\
\hline M. richardii Schwaegr. & $\mathrm{TF}$ & $\mathrm{C}$ & $0-1200 \mathrm{~m}$ & $\begin{array}{l}\text { Neotropical (alcançando } \\
\text { os EUA) e África. Brasil: } \\
\text { AM, BA, MG, ES, RJ, } \\
\text { SP, PR, SC e RS. }\end{array}$ \\
\hline \multicolumn{5}{|l|}{ Orthotrichum sp. } \\
\hline Schlotheimia rugifolia (Hook.) Schwaegr.* & $\mathrm{TF}$ & $\mathrm{C}$ & $0-2700 \mathrm{~m}$ & $\begin{array}{l}\text { Neotropical (alcançando } \\
\text { o sul dos EUA). Brasil: } \\
\text { RO, AM, PA, CE, PE, } \\
\text { BA, GO, MT, MG, ES, } \\
\text { RJ, SP, PR, SC e RS. }\end{array}$ \\
\hline \multicolumn{5}{|l|}{ Polytricaceae } \\
\hline $\begin{array}{l}\text { Atrichum androgynum (Müll. Hal.) A. } \\
\text { Jaeger } \\
\text { POTTIACEAE }\end{array}$ & $\mathrm{TF}$ & $\mathrm{T}$ & $0-800 \mathrm{~m}$ & $\begin{array}{l}\text { Ampla. Brasil: MG, RJ, } \\
\text { PR, SC e RS. }\end{array}$ \\
\hline Barbula agraria $\mathrm{Hedw}$. & $\mathrm{TF}$ & $\mathrm{C}$ & $0-800 \mathrm{~m}$. & $\begin{array}{l}\text { Neotropical. Brasil: AM, } \\
\text { PA, PB, PE, FN, BA e } \\
\text { RJ. }\end{array}$ \\
\hline B. indica (Hook.) Spreng. ex. Steud. & $\mathrm{TF}$ & $\mathrm{R}$ & $0-1200 \mathrm{~m}$ & $\begin{array}{l}\text { Disjunto entre Américas } \\
\text { e Âsia. Brasil: BA, MG, } \\
\text { ES, RJ e SP. }\end{array}$ \\
\hline Hyophila involuta (Hook.) A. Jaeger & $\mathrm{TF}$ & $\mathrm{R}, \mathrm{T}, \mathrm{O}$ & $0-700 \mathrm{~m}$ & $\begin{array}{l}\text { Ampla. Brasil: RO, AM, } \\
\text { PA, PI, PB, BA, GO, ES, } \\
\text { RJ, SP, PR e RS. }\end{array}$ \\
\hline $\begin{array}{l}\text { Leptophascum leptophyllum (Müll. Hal.) J. } \\
\text { Guerra \& M. J. Cano }\end{array}$ & $\mathrm{TP}$ & $\mathrm{T}$ & nível do mar & $\begin{array}{l}\text { Pantropical (ocorrendo } \\
\text { nos EUA e Japão). } \\
\text { Brasil: RJ. }\end{array}$ \\
\hline $\begin{array}{l}\text { RACOPILACEAE } \\
\text { Racopilum tomentosum (Hedw.) Brid. }\end{array}$ & $\mathrm{TP}$ & $\mathrm{R}, \mathrm{T}$ & $0-2700 \mathrm{~m}$ & $\begin{array}{l}\text { América tropical e } \\
\text { subtropical. Brasil: RO, } \\
\text { AC, AM, PA, CE, PE, } \\
\text { BA, DF, GO, MT, MS, } \\
\text { MG, ES, RJ, SP, PR, SC } \\
\text { e RS. }\end{array}$ \\
\hline
\end{tabular}




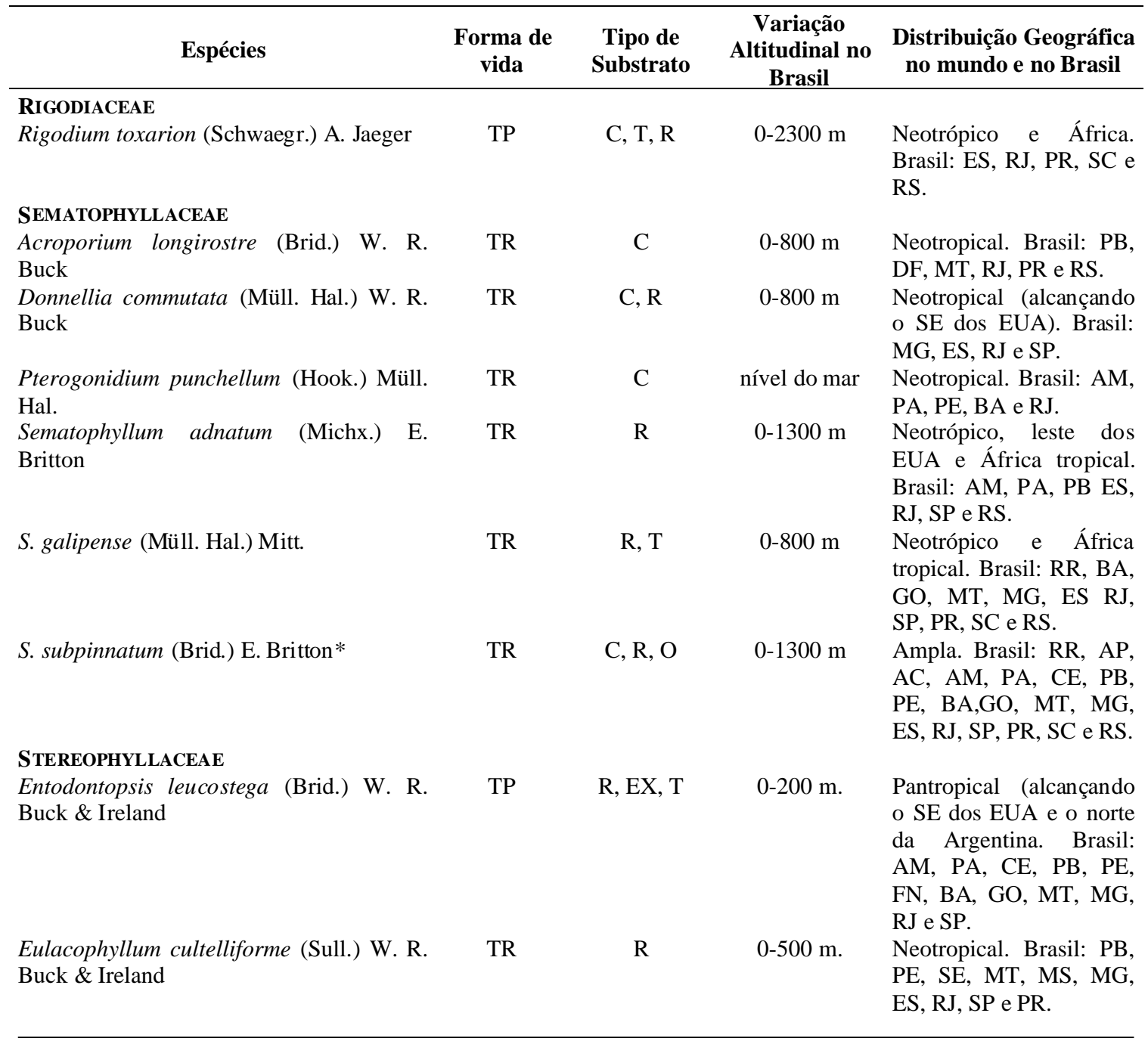

Tabela 2. Comparação entre a brioflora do Arboreto, de Poço das Antas e dos Jardins Botânicos de Belém, espécies em comum entre parênteses.

\begin{tabular}{|c|c|c|c|}
\hline \begin{tabular}{c} 
Grupos \} $\\
{\text { Áreas }}$ & $\begin{array}{l}\text { Arboreto } \\
\text { do JBRJ }\end{array}$ & $\begin{array}{l}\text { Poço das } \\
\text { Antas }\end{array}$ & $\begin{array}{c}\text { Jardins } \\
\text { Botânicos de } \\
\text { Belém }\end{array}$ \\
\hline Hepáticas & 40 & $36(6)$ & $26(3)$ \\
\hline Musgos & 57 & 39 (10) & $54(13)$ \\
\hline Antóceros & 1 & - & - \\
\hline $\begin{array}{l}\text { Total de } \\
\text { espécies }\end{array}$ & 98 & 75 (16) & 80 (16) \\
\hline
\end{tabular}
\end{tabular}

\section{REFERÊNCIAS BIBLIOGRÁFICAS}

Alves, R. J. V. 1992. The flora and vegetation of the Serra São José in Minas Gerais, Brazil. Tese de Doutorado. Botanical Institute of the Czechoslovak Academy of Sciences. Praha, 63p.
Bastos, C. J. P. 2000. Occurrence of some Lejeuneaceae (Jungermanniophyta) in Bahia, Brazil. Tropical Bryology 20: 4554.

, Albertos, B. \& Bôas, S. B. V. 1998. Bryophytes from some Caatinga areas in the state of Bahia (Brazil). Tropical Bryology 14: 69-75.

\& Bôas-Bastos, S. B. V. 1998. Adições à brioflora (Bryopsida) do Estado da Bahia, Brasil. Tropical Bryology 15: 111-116.

\& Bôas-Bastos, S. B. V. 2000. Some New Additions to the Hepatic Flora (Jungermanniophyta) for the State of Bahia, Brazil. Tropical Bryology 18: 1-11. 
Stradmann, M. T. S. \& Bôas-Bastos, S. B. V. 1998b. Additional Contribution to the Bryophyte Flora of Chapada Diamantina National Park, State of Bahia, Brazil. Tropical Bryology 15: 15-20.

\& Yano, O. 1993. Musgos das zonas urbanas de Salvador, Bahia, Brasil.

Hoehnea 20: 23-33.

, Yano, O. \& Bôas-Bastos, S. B. V. 2000.

Briófitas de campos rupestres da Chapada Diamantina, Estado da Bahia, Brasil. Revista Brasileira de Botânica 23: 357-368.

Behar, L., Yano, O. \& Vallandro, C. G. 1992. Briófitas da restinga de Setiba, Guarapari, Espírito Santo. Boletim do Museu de Biologia Mello Leitão, Nova Série, 1: 25-38.

Bischler, H. 1964. Le genre Drepanolejeunea Steph. en Amérique Centrale et Méridionale. Revue Bryologique et Lichénologique 33: 15-179. 1967. Le genre Drepanolejeunea Stephani en Amérique Centrale et Méridionale II. Revue Bryologique et Lichénologique 35: 95-134.

1984. Marchantia L. The New World Species. Bryophytorum Bibliotheca 26: $1-228$.

Bôas, S. B. V. \& Bastos, C. J. P. 1998. Briófitas de uma área de Cerrado no município de Alagoinhas, Bahia, Brasil. Tropical Bryology 15: 101-110.

Bonner, C. E. B. 1953. De Hepaticis III. A contribution to the study of the genus Ceratolejeunea (Spruce) Schiffner. Candollea 14: 163-256.

Bononi, V. L. R. 1989. Recomposição da vegetação da Serra do Mar, em Cubatão, São Paulo, Brasil. Instituto de Botânica. São Paulo, 68p.

Brown, S. \& Lugo, A. E. 1990. Tropical secondary forests. Journal of Tropical Ecology 6: 1-32.

Buck, W. R. 1998. Pleurocarpous mosses of The West Indies. Memoirs of the New York Botanical Garden 82: 400p.
Costa, D. P. 1992. Hepáticas do Pico da Caledônea, Nova Friburgo, Rio de Janeiro, Brasil. Acta Botanica Brasilica 6: 3-39. . 1994. Musgos do Pico da Caledônea, Município de Nova Friburgo, Estado do Rio de Janeiro, Brasil. Acta Botanica Brasilica 8: 141 - 191. . 1999a. Metzgeriaceae (Metzgeriales, Hepatophyta) no Brasil. Tese de Doutorado. Universidade de São Paulo. Instituto de Biociências. São Paulo, 261p. . 1999b. Epiphytic bryophyte diversity in primary and secondary lowland rainforests in southeastern Brazil. The Bryologist 102: 320-326.

\& Yano, O. 1988. Hepáticas talosas do Parque Nacional da Tijuca, Rio de Janeiro, Brasil. Acta Botanica Brasilica 1 (2supl.): 73-82.

\& Yano, O. 1993. Briófitas da Restinga de Massambaba, Rio de Janeiro. Anais III Simpósio de Ecossistemas da Costa Brasileira, Serra Negra 3: 144 - 152.

\& Yano, O. 1995. Musgos do Município de Nova Friburgo, Rio de Janeiro, Brasil. Arquivo do Jardim Botânico do Rio de Janeiro 33: 99-118.

\& Yano, O. 1998. Briófitas da restinga de Macaé, Rio de Janeiro, Brasil. Hoehnea 25: 99-119.

Evans, A. W. 1925. The lobate species of Symphyogyna. Transaction of the Connecticut Academy of Arts and Science 27: 1-50.

Florschütz, P. A. 1964. Mosses of Suriname. Leiden. E. J. Brill, 271p.

Florschütz de - Waard, J. 1986. Flora do Suriname. Musci (Part II). Leiden. E. J. Brill, 273-361.

Fulford, M. H. 1945. Studies on America Hepaticae 6. Ceratolejeunea. Brittonia 5: 368-403.

. 1976. Manual of the leafy Hepaticae of Latin America IV. Memoirs of the New York Botanical Garden 11: 393-535.

Germano, S. R. \& Pôrto, K. C. 1996. Floristic survey of epixylic bryophytes of an area 
remnant of the Atlantic Forest (Timbaúba, PE, Brazil). 1. Hepaticopsida (except Lejeuneaceae) and Bryopsida. Tropical Bryology 12: 21-28.

Giancotti, C. \& Vital, D. M. 1989. Flora briofítica da reserva Biológica da Serra de Paranapiacaba, São Paulo: 1. Lejeuneaceae (Hepaticopsida). Acta Botanica Brasilica 3: 169-177.

Gradstein, S. R. 1981. Miscellanea Hepaticologica 211-220. The Journal of the Hattori Botanical Laboratory 49: 85-92.

. 1994. Lejeuneaceae: Ptychantheae, Brachiolejeuneae. Flora Neotropica. Monograph 62. The New York Botanical Garden. New York, 261p.

\& Buskes, G. M. C. 1985. A revision of Neotropical Archilejeunea (Spruce) Schiffn. Bei. Nova Hedwigia 80: 89-112. , Churchill, S. P. \& Salazar-Allen, N. 2001. Guide to the Bryophytes of Tropical America. Memoirs of the New York Botanical Garden 86: 577p.

, Klein, R., Kraut, L., Mues, R., Spörle, J. \& Becker, H. 1992. Phytochemical and morphological support for the existence of two species in Monoclea (Hepaticae). Plant Systematics and Evolution 180: 115-135.

\& Pócs, T. 1989. Bryophytes. In: Lieth, H. \& Werger, M. J. A. (eds.). Tropical Rain Forest Ecosystems. Elsevier Science Publishers. Amsterdam, 311$325 \mathrm{p}$.

Harley, R. M. (comp.). 1995. Bryophyta. In: Stannard, B. L. (ed.) Flora of Pico das Almas. Chapada Diamantina - Bahia, Brazil. Royal Botanic Gardens. Kew, 803812p.

Hässel de Menéndez, G. G. 1961. Las especies Argentinas del género Symphyogyna. Boletín de la Sociedad Argentina de Botánica 9: 233-260.

1988. A proposal for a new classification of the genera within the Anthocerotophyta. The Journal of the
Hattori Botanical Laboratory 64: 71 86.

1989. Las especies de Phaeoceros (Anthocerotophyta) de América del Norte, Sud y Central; la ornamentación de sus esporas y taxonomia. Candollea 44: 715-739.

Hell, K. G. 1969. Briófitas talosas dos arredores da cidade de São Paulo. Boletim da Faculdade de Filosofia, Ciências e Letras da Universidade de São Paulo, Botânica 25: 1-190.

Herzog, T. 1925. Contribuições ao conhecimento da flora bryológica do Brasil. Archivos de Botanica do Estado de São Paulo 1: 27-105.

Heywood, V. H. 1990. Estratégia dos Jardins Botânicos para a conservação. Jardim Botânico do Rio de Janeiro. 69p. (tradução: The Botanic Gardens conservation strategy).

Hirai, R. Y., Yano, O. \& Ribas, M. E. 1998. Musgos da mata residual do centro politécnico (capão da educação física), Curitiba, Paraná, Brasil. Boletim do Instituto de Botânica de São Paulo 11: 81-118.

Index Seminum pro mutua commutatione offert. 1990. Rio de Janeiro: Jardim Botânico do Rio de Janeiro. 50p.

Jovet-Ast, S. 1993. Riccia L. (Hépatiques, Marchantiales) d'Amérique Latine. Taxons des sous-genres Thallocarpus, Leptoriccia, Ricciella. Cryptog., Revue Bryologique et Lichénologique 14: 219-301.

Lemos-Michel, E. 1980. O gênero Frullania (Hepaticopsida) no Rio Grande do Sul, Brasil. Dissertação de Mestrado. Universidade Federal do Rio Grande do Sul, Porto Alegre. 159p.

. 1983. Frullania (Jungermanniales, Hepaticopsida) no Rio Grande do Sul, Brasil I. Sub-gênero Diastoloba. Revista Brasileira de Botânica 6: 115123.

1999. Briófitas epífitas sobre Araucaria angustifolia (Bert.) Kunze 
no Rio Grande do Sul, Brasil. Tese de Doutorado. Universidade de São Paulo. Instituto de Biociências. São Paulo, 318 p.

Lisboa, R. C. L. 1994. Adições à Brioflora do Estado do Pará. Boletim do Museu Paraense Emílio Goeldi, série Botânica 10: 15 - 42 .

\& Ilkiu-Borges, F. 1995. Diversidade das briófitas de Belém (PA) e seu potencial como indicadoras de poluição urbana. Boletim do Museu Paraense Emílio Goeldi, série Botânica 11: 199-225.

\& Ilkiu-Borges, A. L. 1997. Novas Ocorrências de Bryophyta (musgos) para o Estado do Pará, Brasil. Acta Botanica 27: 81-102.

\& Maciel, U. N. 1994. Musgos da Ilha de Marajó - I - Afuá (Pará). Bol. Boletim do Museu Paraense Emílio Goeldi, série Botânica 10: 43-55. , Muniz, A. C. M. \& Maciel, U. N. 1998. Musgos da Ilha de Marajó - III - Chaves (Pará). Boletim do Museu Paraense Emílio Goeldi, série Botânica 14: 117125.

\& Yano, O. 1987. Novas ocorrências de briófitas na Amazônia brasileira. Boletim do Museu Paraense Emílio Goeldi, série Botânica 3: 141-156.

Mägdefrau, K. 1982. Life forms of bryophytes. In Smith, A. J. E. (ed.) Bryophyte Ecology. New York. 45-58p.

Martins, D. V., Bastos, C. J. P. \& Yano, O. 1990. Ocorrência de Notothylas vitalii e Phaeoceros laevis em São Paulo e Bahia, Brasil. Revista Brasileira de Botânica 13: $15-18$.

Montfoort, D. \& Ek, R. C. 1990. Vertical Distribution and Ecology of Epiphytic Bryophytes and Lichens in a Lowland Rain Forest in French Guiana. Institute of Systematic Botany. Utrecht, $56 \mathrm{p}$.

Oliveira, S. M. 2001. Ecologia de Populações de Três Espécies de Musgos Acrocárpicos em Remanescentes de Floresta Atlântica, Recife-Pernambuco-
Brasil. Dissertação de Mestrado. Universidade Federal de Pernambuco. Centro de Ciências Biológicas, 52 p.

Oliveira e Silva, M. I. M. N. 1998. Briófitas da Reserva Ecológica de Rio das Pedras, município de Mangaratiba, do Parque Estadual da Ilha Grande e da Reserva Biológica Estadual da Praia do Sul, município de Angra dos Reis, estado do Rio de Janeiro. Tese de Doutorado. Instituto de Biociências, Universidade de São Paulo, 321 p.

Pôrto, K. C. 1990. Bryoflores d'une forêt de plaine et d'altitude moyenne dans l'état de Pernambuco (Brésil): Analyse floristique. Cryptogamie, Bryologique et Lichénolique 11: 109-161.

\& Bezerra, M. F. A. 1996. Briófitas da Caatinga. 2. Agrestina, Pernambuco, Brasil. Acta Botanica Brasilica 10: 93 102.

, Gradstein, S. R., Yano, O., Germano, S. R. \& Costa, D. P. 1999. New or interesting reccords of Brazilian bryophytes. Tropical Bryology 17: 3945.

\& Oliveira, S. M. 2000. New records of bryophytes for Permanbuco State, Brazil. Tropical Bryology 18: 107-114. \& Yano, O. 1998. Ocorrências novas de briófitas para o Brasil. Revista Brasileira de Botânica 21: 125-134.

Rao, D. N. 1982. Responses of Bryophytes to Air Pollution. In: Smith, A. G. E. (ed.) Bryophyte Ecology. New York, 445-472p.

Rebelo, C. F., Struffaldi-De, V. Y. \& Domingos, M. 1995. Estudo ecológico de comunidades de briófitas epífitas na Reserva Biológica de Paranapiacaba, SP, em trechos de floresta sujeitos à influência da poluição aérea. Revista Brasileira de Botânica 18: 1-16.

Reese, W. D. 1993. Calymperaceae. Flora Neotropica. Monograph 58. The New York Botanical Garden. New York, 102p.

Reiner-Drehwald, M. E. \& Goda, A. 2000. Revision of the genus Crossotolejeunea 
(Lejeuneaceae, Hepaticae). The Journal of the Hattori Botanical Laboratory 89: $1-54$.

Richards, P. W. 1984. The Ecology of Tropical Forest bryophytes. In Schuster, R. M. (ed.) New Manual of Bryology. Hattori Botanical Laboratory, vol. 2, 1233-1270p.

Robbins, R. G. 1952. Bryophyte ecology of a dune area in New Zealand. Vegetatio, Acta Geobotanica 4: 1-31.

Sá, P. S. A. \&. Pôrto, K. C. 1996. Novos registros de Hepaticopsida (Bryophyta) para Pernambuco. Revista Nordestina de Biologia 11: 37-43.

Sampaio, A. J. 1916. A Flora de Mato Grosso. Archivos do Museu Nacional do Rio de Janeiro 19: 1-125.

Santiago, R. L. 1997. Estudos Brioflorísticos de três formações vegetais no município de Bonfim-Roraima. Dissertação de Mestrado. Universidade Federal de Pernambuco. Recife, 124p.

Schäfer-Verwimp, A. 1989. New or interesting records of Brazilian Bryophytes, II. The Journal of the Hattori Botanical Laboratory 67: 313-321.

1991. Contribution to the knowledge of the bryophyte flora of Espírito Santo, Brazil. The Journal of the Hattori Botanical Laboratory 69: 147-170. . 1996. New or interesting records of Brazilian Bryophytes, V. Candollea 51: 283-302.

\& Giancotti, C. 1993. New or interesting records of Brazilian Bryophytes, IV.

Hikobia 11: 285-292.

\& Vital, D. M. 1989. New or interesting records of Brazilian Bryophytes. The Journal of the Hattori Botanical Laboratory 66: 255-261.

Schiffner, V. \& Arnell, S. 1964. Ergebnisse der botanischen Expedition der kaiserlichen Akademie der Wissenschaften nach Südbrasilien 1901. II. Hepaticae. Österrichische Akademie der Wissenschaften,
Mathematisch-Naturwissenschaftliche Klasse, Denkschriften 111: 1-156.

Schuster, R. M. 1980. Evolution, phylogeny and classification of the Hepaticae. In Schuster, R. M. (ed.). New Manual of Bryology. Japan, Hattori Botanical Laboratory. vol. 2, 892-1070p.

Sergio, C. 1981. Alterações da flora briológica epifítica na área urbana de Lisboa, nos últimos 140 anos. Boletim da sociedade broteriana, série 2, 54: 313-331.

Sharp, A. J., Crum, H. \& Eckel, P. M. 1994. The Moss Flora of Mexico. Memoirs of the New York Botanical Garden 69: $1113 p$.

Spruce, R. M. 1884-1885. Hepaticae Amazonicae et Andinae. Transaction and Proccedings of the Botanical Society Edinburgh 15: 1-588.

Stotler, R. E. 1969. The genus Frullania subgenus Frullania in Latin America. Nova Hedwigia 18: 397-555. , Salazar Allen, N., Gradstein, S. R., McGuinness, W., Whittemore, A. \& Chung, C. 1999. A Checklist of the Hepatics and Anthocerotes of Panamá. Tropical Bryology 15: 167-195.

Vianna, E. C. 1970. Marchantiales e Anthocerotales coletadas no Rio Grande do Sul. Iheringia. Botanica 14: 45-54. . 1976. Marchantiales (Hepaticopsida) coletadas no Rio Grande do Sul. Tese de Livre Docência. Universidade Federal do Rio Grande do Sul. Porto Alegre, 226p. . 1981. Sobre a ocorrência nova de Monoclea Hook. (Hepaticae) no Rio Grande do Sul, Brasil. Iheringia, Botanica 26: 165-167. . 1985. Marchantiales. Boletim do Instituto de Biociências de Porto Alegre 37: 1-213.

Visnadi, S. R. 1998. Briófitas em Ecossistemas Costeiros do Núcleo Picinguaba do Parque Estadual da Serra do Mar, Ubatuba - SP. Tese de Doutorado. Universidade Estadual Paulista. Rio Claro, 174p. 
\& Vital, D. M. 1989. Briófitas rupícolas de um trecho do rio Bethary, Yporanga, Estado de São Paulo. Acta Botanica Brasilica 3: 179-183.

Vital, D. M. 1980. Erpodiaceae (Musci) do Brasil. Tese de Mestrado. Universidade Estadual de Campinas. São Paulo, 135p. , Giancotti, C. \& Pursell, R. A. 1991. The bryoflora of Fernando de Noronha, Brazil. Tropical Bryology 4: 23-24.

Vital, D. M. \& Visnadi S. R. 1994. Bryophytes of Rio Branco Municipality, Acre, Brazil. Tropical Bryology 9: 69-74.

Vitt, D. H. 1984. Classification of the Bryopsida. In: Schuster, R. M. (ed.). New Manual of Bryology. Hattori Botanical Laboratory. vol. 2, 696-759p.

Yano, O. 1984a. Checklist of Brazilian liverworts and hornworts. The Journal of the Hattori Botanical Laboratory 56: 481-548. 1984b. Briófitas. In Fidalgo, O. \& Bononi, V. L. R. (coord.) Técnicas de coleta, herborização e preservação de material botânico. Instituto de Botânica. São Paulo, 62p. (manual no.4).

. 1987. Addittions to the Brazilian Hepaticae. The Bryologist 90: 374-375. .1989. An additional checklist of Brazilian bryophytes. The Journal of the Hattori Botanical Laboratory 66: 371-434. 1993. Briófitas do nordeste brasileiro: Estado da Paraíba, Brasil. Biologia Brasilica 5: 87-100.

. 1994. Briófitas da Serra de Itabaiana, Sergipe, Brasil. Acta Botanica Brasilica 8: 45- 57.

. 1995. A new additional annotated checklist of Brazilian bryophytes. The Journal of the Hattori Botanical Laboratory 78: 137-182.

1996. A checklist of Brazilian bryophytes. Boletim do Instituto de Botânica de São Paulo 10: 47-232.

\& Andrade-Lima, D. 1987. Briófitas do nordeste brasileiro: Estado de Pernambuco. Revista Brasilleira de Botânica 10: 171-181.
\& Colletes, A. G. 2000. Briófitas do Parque Nacional de Sete Quedas, Guaíra, PR, Brasil. Acta Botanica Brasilica 14: 127-242.

\& Costa, D. P. 1992. Novas ocorrências de briófitas no Brasil. Anais do $8^{\circ}$ Congresso Sociedade Botânica de São Paulo, Campinas. São Paulo, 33-45p.

\& Costa, D. P. 2000. Flora dos Estados de Goiás e Tocantins. Criptógamas: Briófitas. vol. 5, 33p.

\& Lisboa, R. C. L. 1988. Briófitas do território Federal do Amapá, Brasil. Boletim do Museu Paraense Emílio Goeldi, Série Botânica 4: 243-270.

\& Mello,Z. R. 1992. Briófitas novas para o Estado de Roraima, Brasil. Acta Amazonica 22: 23-50.

\& Santos, S. X. 1993. Musgos da gruta de Mirassol, São Paulo. Acta Botanica Brasilica 7: 89-106.

Yuzawa, Y. 1988. Some little-known species of Frullania subg. Diastoloba described from Latin America. The Journal of the Hattori Botanical Laboratory 64: 437 449.

. 1991. A monograph of subg. Chonanthelia of gen. Frullania (Hepaticae) of the world. The Journal of the Hattori Botanical Laboratory 70: 181-291.

\& Koike, N. 1989. Studies on the type specimens of Latin American Frullania species. The Journal of the Hattori Botanical Laboratory 66: 343-358. 